\title{
Una mirada feminista a la comunidad arqueológica peruana
}

\section{A feminist perspective on the Peruvian archaeological community}

\author{
Carito Tavera Medina ${ }^{1}$ \\ Universidad Nacional Mayor de San Marcos. Lima, Perú \\ caritotaveramedina@gmail.com \\ ORCID 0000-0001-6387-4360
}

Citar como: Tavera, C. (2019). Una mirada feminista a la comunidad arqueológica peruana. Desde el Sur, 11(2), pp. 239-260.

\section{RESUMEN}

Las experiencias de vida de mujeres, hombres y diversidades (comunidad LGTBIQ+) en un país como el Perú siguen estando sumergidas en un universo de desigualdades sociales intersectadas por factores como clase social, origen étnico, género, sexo, edad; por solo mencionar algunos de ellos. Dichas asimetrías sociales se ven expresadas en los distintos espacios de la sociedad, incluido el mundo académico de las ciencias sociales y humanidades (Vargas, 2014; Alcázar y Balarin, 2018) espacio en el que arqueólogas y arqueólogos interactuamos y del cual formamos parte. Este artículo busca poner sobre el tapete un tema poco o nada discutido al interior de nuestra comunidad profesional: la violencia de género. Ello como una primera iniciativa de sensibilización a la comunidad arqueológica peruana.

\section{PALABRAS CLAVE}

Feminismo, arqueología, violencia de género, arqueología feminista

\footnotetext{
1 Carito Tavera Medina es arqueóloga, egresada de la Universidad Nacional Mayor de San Marcos. Ha sido becaria de la Unesco (2014) y Fondecyt Perú (2016-2018). Es magíster por la Université de Rennes, Francia, y especialista en arqueobotánica. Sus investigaciones arqueológicas giran en torno al estudio de las implicancias sociales de los rituales funerarios de la sociedad moche. Como arqueóloga feminista, se ocupa de estudiar y visibilizar la historia y los aportes femeninos a la arqueología peruana, así como abordar la problemática de sus relaciones de género.
} 


\section{ABSTRACT}

The life experiences of women, men and minorities (the LGBTIQ + community) in a country like Peru continue to find themselves submerged in a universe of social inequalities, intersected by factors such as social class, ethnic origin, gender, sexuality and age, to mention just a few. These social asymmetries are expressed across different social spaces, including the academic world and the fields of social sciences and the humanities (Vargas, 2014; Alcazar \& Balarin, 2018), in which male and female archaeologists interact, and of which we form a part. This article seeks to shed light upon an issue that is discussed rarely, if at all: gender violence. The aim is to provide a first step towards the raising of awareness within the Peruvian archaeological community.

\section{KEYWORDS:}

feminism, archaeology, gender violence, feminist archaeology

\section{Introducción}

El feminismo como movimiento político y social ha brindado a las mujeres y minorías las herramientas para luchar por la reivindicación de sus derechos. Se pueden mencionar logros como el sufragio femenino y el derecho a la participación política, el derecho a la educación básica regular, el acceso a las aulas universitarias, el ejercicio profesional, entre otros. Todo ello hace «indudable que el movimiento feminista ha sido, en nuestro país, un protagonista de los cambios vividos [en relación con] las mujeres y su situación de exclusión, y en la lucha por la democracia» (Vargas, Orving y Trapaso, 2004, p. 3).

Como señala el PNUD (Informe sobre Desarrollo Humano, 2010) la desigualdad basada en el género es un obstáculo para el desarrollo de los individuos y su nación. En el caso del Perú, la marginalidad social en la que aún operan las mujeres al interior de nuestra sociedad se refleja en las tasas de analfabetismo y deserción estudiantil de niñas en la educación básica regular, el desempleo e informalidad laboral de mujeres, el alto número de casos de violencia física y psicológica, etc. Estas desventajas que afrontan las mujeres son documentadas por medio del índice de desigualdad de género (IDG), que en el caso del Perú ha ido en descenso de 2000 a 2015, momento inicial en el cual se reportaba un 0,526 en comparación con un 0,420 al interior de una escala que va del 0 (no existe desigualdad) a 1 (se da una desigualdad completa) (INEI, 2016). 
Entre algunos de los indicadores considerados para la lectura de la disminución de la desigualdad de género se encuentra la autonomía de las mujeres $^{2}$, el acceso a servicios de salud y educación (INEI, 2016, pp. 17-39 y 57-105). En 2015 la población de mujeres peruanas ascendía a 15545 800 , que representan del $49,9 \%$ de nuestra población (INEI, 2016). De este universo de mujeres se registró que el $32,3 \%{ }^{3}$ carecían de ingresos económicos propios, un $70,8 \%$ accedía a servicios de salud pública ${ }^{4}$ y se ha reportado un 9,0\% de mujeres analfabetas, en comparación con el 3,0\% de hombres. A todo ello, debemos sumar la situación de vulnerabilidad sobre la autonomía física de las mujeres, la cual se ha materializado en 823 víctimas de feminicidios ${ }^{5}$ reconocidos por el Observatorio de Criminalidad del Ministerio Público de 2009 a 2015; y un total de 32,0\% ${ }^{6}$ de mujeres de 15 a 49 años que han sufrido algún tipo de violencia física o sexual por parte de sus parejas en algún momento de sus vidas de acuerdo con las entrevistas procesadas en 2015.

Este tipo de acciones que atentan contra los derechos básicos de las mujeres son denominados violencia de género ${ }^{7}(\mathrm{ONU}, 1995)$ y «se refiere a la violencia que se ejerce contra las mujeres por el hecho de ser mujeres. Esto es, todas las formas de violencia que perpetúan el control sobre las mujeres, o que imponen o restablecen una condición de sometimiento para las mujeres» (Castro, 2016, p. 340). Las estadísticas en el Perú señalan que la violencia física se da de forma más recurrentes en los sectores económicos bajo y medio; sin embargo, es posible que en la población de clase media y alta los actos de violencia sean más recurrentes y de carácter psicológico (INEI, 2016, p. 123).

Este tipo de violencia de carácter social es un mecanismo que se basa en ciertos tipos de relaciones y procesos sociales de desigualdad y

2 Para ello se toma en consideración la autonomía física, económica y la toma de decisiones; todo ello amparado en la X Conferencia Regional Sobre la Mujer de América Latina y el Caribe (2007) (INEI, 2016)

3 La estadística consideró a la población a partir de los 14 años a más. Es importante considerar que el $12,5 \%$ de hombres peruanos de 14 a más no cuenta con ingresos económicos propios, lo cual evidencia una situación abismal de posibilidades al interior de la población. 4 Dentro de esta estadística se consideró la cobertura por el SIS y EsSalud (INEI, 2016, gráfico 4.5).

5 Asimismo, por medio de los datos del Ministerio Público ha podido registrar las modalidades empleadas en los feminicidios entre las que se reportan de forma reincidente de forma ascendente a descendente: el acuchillamiento, la asfixia, los golpes, ser baleada, el envenenamiento, ser quemada y el desbarrancamiento (INEI, 2016, p. 22).

6 Datos obtenidos por medio de la Encuesta Demográfica y de Salud Familiar, que en consideración del INEI «proveen un marco de entrevista más seguro y confiable, en el que el tema de la violencia puede ser abordado junto con otros de carácter confidencial como los relacionados con la salud reproductiva» (INEI, 2016, p. 120).

7 Este tipo de violencia puede ser de carácter físico, sexual o psicológico (Convención Interamericana para la Prevención, Sanción y Erradicación de la Violencia contra la Mujer). 
opresión histórica, donde la dominación masculina estructurada busca mantener el control y poder, situación propia de la estructura patriarcal (Hunnincut, 2009; Castro, 2016). El Ministerio de la Mujer y Poblaciones Vulnerables del Perú (MIMP, 2016) señala que la violencia de género ${ }^{8}$ responde a una violencia estructural presente en nuestra sociedad, en la cual se discrimina y desvaloriza todo aquello relacionado con lo femenino.

EI MIMP señala que una de las modalidades más visibles en que se presenta la violencia de género es en el ámbito laboral por medio del hostigamiento o acoso sexual. En 2009 el Ministerio del Interior recepcionó un total de 3399 quejas sobre hostigamiento sexual, todas ellas interpuestas por mujeres (MIMP, 2016, p. 36). Una encuesta desarrollada por APTITUS en 2015 determinó que el 19\% de mujeres peruanas han sufrido actos de hostigamiento sexual en sus espacios de trabajo, mientras que la Superintendencia Nacional de Fiscalización Laboral (Surafil) identificó, de 2017 a 2018, un total de 64 casos de hostigamiento sexual ${ }^{9}$ en las áreas laborales del sector público (OEFA, 2018).

A partir de este panorama general sobre las condiciones sociales que afrontan las mujeres en el Perú, nos preguntamos si estas asimetrías se ven reproducidas en el espacio académico de las ciencias sociales y humanidades. Un primer punto a tomar en cuenta es el que nos brinda Graeber, antropólogo anarquista, quien señala a las universidades como instituciones que "fomentan [...] el sectarismo, las divisiones entre "escuelas", y las lealtades de séquitos frente a quienes emergen como luminarios cuan santos o señores feudales [y son] las universidades las únicas instituciones más o menos intactas desde la Edad Media hasta hoy» (Graeber, 2005, p. 194, en Anderson, 2014, p. 31). De esta forma, las universidades se configuran como espacio propicio de legitimización del poder y aprendizaje de rituales académicos que permitirán el surgimiento y la creación de referentes a seguir por parte de los estudiantes y futuros profesionales, característica que debemos tomar en consideración y que cobrará suma importancia en los próximos párrafos.

Mientras que en otras latitudes (Estados Unidos y Europa) desde la década de 1970 la asimilación del feminismo al mundo académico acarreó la crítica epistemológica a la generación de conocimiento científico (Arrieta de Guzmán, 2018), la reflexión interna sobre las dinámicas de reproducción

8 Este tipo de violencia puede ir desde la violencia que se da en el ámbito conyugal (privado) hasta la violencia homofóbica, hasta llegar a cometer «crímenes de odio» (MIMP, 2016). 9 Estos son aquellos que llegaron a ser reportados y denunciados, ya que existen muchísimos que son ocultados por miedo de las denunciantes a ser estigmatizadas, revictimizadas, discriminadas e incluso despedidas por efectuar la denuncia. 
de la academia y las relaciones de poder existentes, en el Perú este proceso fue paulatino (Vargas et al., 2004). Las ciencias sociales, como la historia, la antropología y la sociología, han brindado los primeros aportes para visibilizar a las mujeres en la historia del Perú (Zegarra, 1999; Andreo y Guardia, 2002; Mannarelli, 2018; Lauro, 2019). Sin embargo, un campo en pleno auge es el que aborda las problemáticas y desafíos que afrontan las mujeres al incorporarse al mundo académico peruano (González Moreno, 2012).

En el campo de las ciencias sociales destaca el proyecto emprendido por el Grupo Sofia, que ha desarrollado una serie de estudios del estado de la cuestión de las mujeres en este ámbito de las ciencias (Vargas, 2014; Alcázar y Balarin, 2018). Como menciona Balarin, el Perú ha experimentado un proceso de feminización de las aulas universitarias; a pesar de ello, «las mujeres están marcadamente subrepresentadas, tanto en su presencia en cargos académicos y de liderazgo, como en la visibilidad del conocimiento que producen» $(2014$, p. 50).

Ella nos invita a reflexionar sobre el impacto de las diferencias entre hombres y mujeres en términos salariales, contractuales, roles en el hogar y carga horaria, así como el rol del Estado en el desarrollo de políticas de equidad de género. En relación con este último punto, Janina León (2014) pone en evidencia, a partir de los datos recabados por el Censo Universitario (2010), la existencia de una preponderancia de contratación docente masculina tanto en universidades públicas como privadas en el área de las ciencias sociales. Si consideramos estos aportes en el contexto brindado por Graeber, sobre el papel que juega la universidad para la reproducción de la academia, resulta de suma importancia reflexionar sobre las estructuras, estrategias y dinámicas de poder existentes en la academia, y a partir de ello emprender un proyecto en aras de un ambiente académico con igualdad de posibilidades y libre de violencia de género.

En esta dirección e impulsadas por el desarrollo de una arqueología crítica, hemos visto conveniente enmarcar nuestra investigación desde el enfoque del pensamiento feminista.

\section{La arqueología feminista y su crítica a la academia}

El feminismo o, mejor dicho, los feminismos son considerados «movimientos sociales, como práctica política y como disciplina que se enseña; tienen una historia, una praxis propia y un caudal de presupuestos epistemológicos que se alimentan día con día conforme se desarrolla su pensamiento y su práctica, misma que se construye constantemente de acuerdo con el contexto en que se desenvuelven las mujeres que se autodefinen como feministas» (Jaiven, 2016, p. 139). En el caso de la arqueología, el pensamiento feminista posee una historia y praxis propia, que fue introducido por Conkey y Spector (1994) por primera vez. 
De acuerdo con Rubí Gómez Campos (2013, p. 84, en Arrieta de Guzmán, 2018, p. 53), «el objetivo de la teoría feminista es superar la parcialidad masculinazante de las ciencias y la filosofía "neutral"». Es así como el empirismo feminista se propone eliminar los rasgos sexistas de la investigación científica y abogar por ejercer cambios en el lenguaje empleado en la producción de conocimiento. Asimismo, la epistemología del punto de vista feminista resalta que las ciencias tradicionales poseen una fuerte carga sexista y androcéntrica, y aboga por la inclusión del punto de vista femenino y de los marginados con el objetivo de generar una actitud reflexiva de las otredades sociales, lo que permite el enriquecimiento de la agenda de investigación (Arrieta de Guzmán, 2018). La arqueología feminista se nutre de estas posturas y se configura como una postura «radical, pero no rompe con la tradición científica occidental» (Berrocal, 2009, p. 33) y que cohabita desde un activismo político con las llamadas arqueología indigenista y arqueología poscolonial, espacios desde donde se busca dar voz a las marginalidades arqueológicas (Conkey, 2005).

La tradición arqueológica norteamericana hizo «oficial» el feminismo como corriente arqueológica, buscando no solo visibilizar a las mujeres desde el registro arqueológico, sino reconfigurar la manera de hacer arqueología a nivel epistemológico ${ }^{10}$ y reconfigurar las dinámicas internas de la práctica arqueológica (Berrocal, 2009, p. 27). Engendering Archaeology: Women and Prehistory (Gero y Conkey, 1991) marca un hito en la arqueología feminista, al hacer énfasis en tres puntos: el machismo intrínseco en la investigación arqueológica, la ausencia de las mujeres en el registro arqueológico y la falta de un enfoque de género al analizar las sociedades.

Desde esta visión crítica hemos visto conveniente ahondar en las dinámicas internas de la academia arqueológica (Conkey y Wylie, 1998; She, 2000; Claasen, 2000). Uno de los tópicos que evidencia el androcentrismo de nuestra academia es la construcción de su historia. Para ello, basta con detenernos en los casos de invisibilización histórica que reportan Colin Renfrew y Paul Bahn (2001, pp. 36-37) al señalar lo siguiente: «The story of many early women archaeologist was one of exclusion and lack of recognition or promotion - or even employment. Furthermore, many brilliant academic women accept that, after marriage, their career would no longer be professional one, and supported the academic work of their husband with little public recognition».

10 Se busca cambiar la forma en que nuestras categorías de análisis arqueológico operan tradicionalmente, al buscarse primero introducir como sujeto cognoscible o sujeto histórico a las mujeres, y reconstruir las condiciones históricas de inequidad, relaciones de poder y las distintas realidades existentes al interior de las sociedades vistas desde la óptica del género (Conkey y Spector, 1984; Conkey y Gero, 1997). 
A modo de respuesta han surgido múltiples aportes sobre la vida y obra de las mujeres en la historia de la arqueología, como el volumen editado por Díaz Andreu y Sorensen (1998), por solo mencionar un ejemplo.

En lo que respecta al campo de la arqueología peruana y la construcción de las historias de las mujeres al interior de la disciplina, se constata una producción literaria bastante limitada (Novoa, 2013; Lavallée, 2013). Como lo han señalado Díaz-Andreu (2013) y Patterson (2013), es innegable la ausencia de las mujeres en la construcción histórica de la arqueología peruana y nos invitan abrir un nuevo acápite en la agenda de la investigación.

Una de las primeras iniciativas que recoge estas reflexiones es el trabajo presentado por Tavera y Santana (2018), donde se aborda la participación de las mujeres en los espacios de difusión académica en la arqueología peruana (también ver los aportes de Santana, Cabrera, Arroyo y Pacheco en este número). Por medio de su estudio, las autoras resaltan la infrarrepresentación de las mujeres en el ámbito de producción científica peruana, y destacan que de un total de 708 artículos $^{11}$ solo se registraron 198 autoras, en contraste con la participación de 508 autores. Las autoras rescatan también la importancia que adquiere la posición en la que se encuentran los investigadores, hombres y mujeres, al momento de publicar un trabajo colaborativo. Si bien los datos arrojaron que la participación femenina va en aumento, todavía componen una minoría como primeras, segundas y terceras autoras. Finalmente, las autoras recalcan que, aunque el género es uno de los factores a tomar en cuenta sobre los resultados obtenidos, que se suma a las reducidas y focalizadas políticas de fomento de la participación de las mujeres en la ciencia, esta realidad es de carácter multicausal y se sugiere detenerse a analizar los contextos sociales y las vivencias en los que se enmarcan las arqueólogas peruanas.

En esta misma dirección, y tras esta primera experiencia, motivadas por lo propuesto por Alison Wylie (2007), nos hemos enfocado en rescatar y dar un primer panorama sobre las experiencias de las mujeres al interior de la arqueología peruana.

\section{Metodología}

Nuestras tribus académicas nos sumergen en micromundos dotados de una serie de idiosincrasias acumulativas, las cuales han sido construidas a lo largo de más de 100 años, en el caso de la arqueología peruana. La patriarcalidad innata e históricamente construida desde Uhle y Tello

11 Las revistas tomadas en cuenta para la realización de esta investigación fueron el Boletín de Arqueología PUCP y la revista Arqueología y Sociedad de la Universidad Nacional Mayor de San Marcos. En ambos casos se consideraron las publicaciones emitidas hasta 2018. 
nos establecen parámetros y modelos a seguir, que asimilamos a lo largo de nuestro pregrado y se reproduce durante nuestro ejercicio profesional, muchas veces renutriéndose.

Teniendo en cuenta este primer enunciado y contemplando la preponderancia y dominación masculina en el campo de la arqueología (Gero, 1985, 1994) nos propusimos: 1) demostrar la existencia de esta al interior de la arqueología peruana, y 2) identificar comportamientos enmarcados en el concepto de violencia de género.

Para tal objetivo, hicimos uso de dos fuentes: 1) la encuesta \#MeTooArqueología (figura 1), y 2) la encuesta «Hablemos de arqueología» (figura 1). La primera recabó información de carácter testimonial anónimo y se enfocó en generar un espacio para mujeres que sufrieron o evidenciaron experiencias de carácter sexista. La segunda fue mucho más amplia y buscó recabar información diversa que nos diera luces sobre la idiosincrasia de los arqueólogos peruanos, al abordar temas económicos, educativos y de enfoque de género.
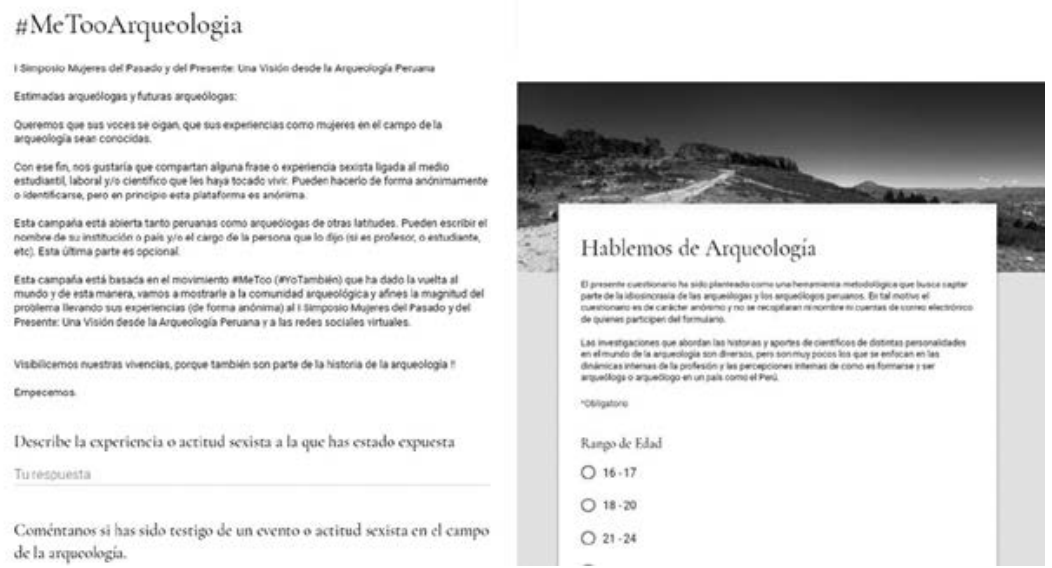

FIGURA 1. Encuesta de la campaña \#MeTooArqueología (simposio «Mujeres del pasado y del presente: una visión desde la arqueología peruana», de 2019) y formulario de la encuesta «Hablemos de arqueología» (Tavera, 2019).

Si bien la iniciativa de \#MeTooArqueología nació con el motivo de visibilizar los comportamientos de corte sexista al interior de la arqueología peruana y presentar los testimonios en el marco del simposio «Mujeres del pasado y del presente: una visión desde la arqueología peruana», estos datos se mantuvieron en reserva. Esto se debió al nivel de detalle de los relatos, que podrían permitir a los agresores identificar a las víctimas y colocarlas en una situación de vulnerabilidad. Por ese motivo, en este documento haremos uso limitado de esta fuente, la cual nos permitió conocer un total de 35 respuestas en torno a las siguientes preguntas: 
a) Describe la experiencia o actitud sexista a la que has estado expuesta, y b) Coméntanos si has sido testigo de un evento o actitud sexista en el campo de la arqueología.

La encuesta "Hablemos de arqueología» estuvo compuesta por 23 preguntas de carácter anónimo y se contó con la participación de 55 personas. Debido a los intereses de esta publicación, nos enfocaremos solo en ocho preguntas, que analizaremos a partir del rango de edad del participante, su sexo y la universidad donde cursó el pregrado en Arqueología.

Las preguntas contempladas fueron las siguientes:

a. ¿Consideras que la experiencia de hombre, mujeres y diversidades (comunidad LGTBI+) es homogénea al interior de la arqueología peruana?

b. ¿Has sentido que el ambiente de la arqueología peruana puede llegar a ser hostil con las mujeres?

c. ¿Has presenciado (o por medios digitales) el uso de lenguaje ofensivo o deplorable hacia una mujer al interior de la arqueología peruana?

d. ¿Has sido víctima de agresión verbal de carácter sexista al interior de la arqueología peruana?

e. ¿Consideras que el hostigamiento sexual es un tema que debe ser tratado al interior de la arqueología peruana?

f. ¿Crees que el hostigamiento sexual se da al interior de la arqueología?

g. ¿Has vivido alguna de las siguientes situaciones? (las opciones a seleccionar respondían a aquellas acciones que tipifican como hostigamiento sexual de acuerdo con la Ley Universitaria actual).

h. Finalmente, ¿consideras que las mujeres experimentan la formación y práctica arqueológica de igual forma que los hombres?

\section{Resultados}

El universo de participantes de la encuesta «Hablemos de arqueología» fue de 55 personas, de las cuales 27 eran del sexo masculino y 27 del sexo femenino. Solo un participante se abstuvo de dar una respuesta. En lo que respecta al rango etario de los encuestados, un 38,2\% tenía entre 25 y 30 años, un 32, 7\% oscilaba entre los 21 y 24 años, el 16,4\% estaba en el rango de los 31-35, mientras que en menor proporción se encontraron los grupos etarios de 36-40 años (7,3\%), 18-20 años (3,6\%) y, por último, un 1,8\% correspondiente a las personas de 41 a 45 años.

En la encuesta participaron estudiantes y egresados de diversas universidades, como la Pontificia Universidad Católica del Perú, la Universidad Nacional Mayor de San Marcos, la Universidad Nacional Federico Villareal, la Universidad Nacional Pedro Ruiz Gallo, la Universidad Nacional de Trujillo, la Universidad Nacional San Cristóbal de Huamanga y la Universidad Nacional San Luis Gonzaga de Ica. 
A la primera pregunta ( ¿ ¿Consideras que la experiencia de hombre, mujeres y diversidades [comunidad LGTBI+] es homogénea al interior de la arqueología peruana?»), un mayoritario $60 \%$ consideró que las experiencias son diferenciadas. Esta respuesta encuentra una especial correspondencia en torno al potencial nivel de hostilidad hacia las mujeres en el ambiente arqueológico, ya que se reportó que un $69,1 \%$ de encuestados afirman la existencia de esta situación. Sin embargo, al separar las respuestas por sexo las respuestas cambian: mientras que un $40 \%$ de hombres niegan la existencia de esta situación, un reducido $18,5 \%$ de mujeres se suman a este tipo de respuesta (figura 2).
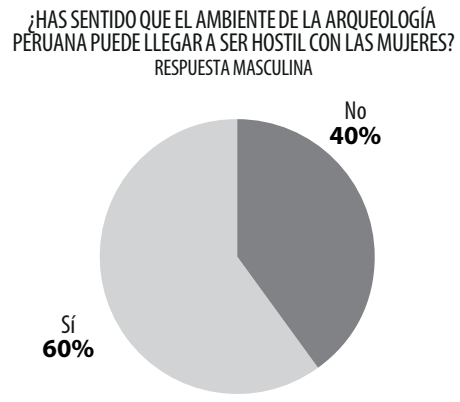

¿HAS SENTIDO QUE EL AMBIENTEDE LA ARQUEOLOGÍA PERUANA PUEDE LLEGARA SER HOSTIL CON LAS MUJERES? RESPUESTA FEMENINA

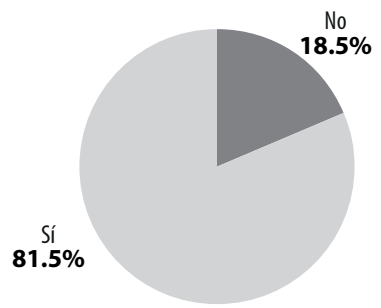

FIGURA 2. De izquierda a derecha. El primer cuadro muestra los resultados masculinos, mientras que el segundo indica las respuestas femeninas a la pregunta que aborda la existencia de un ambiente hostil hacia las mujeres al interior de la arqueología peruana.

En relación con el uso de lenguaje ofensivo hacia una mujer en el marco de la arqueología, considerando que el acto se dio de forma presencial o por medio de medios digitales, un $65,0 \%$ de hombres admite haber sido testigo de este tipo de sucesos, mientras las mujeres lo reportan en menor medida $(51,9 \%)$. Esto nos dice que más de la mitad de los encuestados han vivido de manera física o digital el uso de lenguaje deplorable contra una mujer al interior de nuestra comunidad. En contraste a estos resultados, cuando se les pregunta a las encuestadas si han sido víctimas de agresión verbal de corte sexista al interior de la arqueología peruana, solo un $37,0 \%$ de ellas admite haber pasado por esta circunstancia, mientras que un $20 \%$ de los hombres dice haber sido víctima de esta situación (figura 3). 
¿HAS SIDO VÍCTIMA DE AGRESIÓN VERBAL DE CARÁCTER SEXISTA

EN EL INTERIOR DE LA ARQUEOLOGÍA PERUANA? RESPUESTA FEMENINA

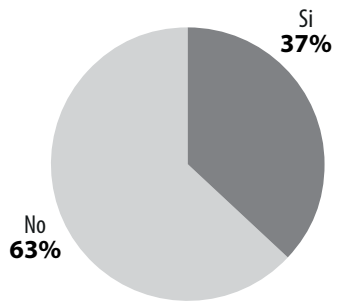

¿HAS SIDO VÍCTIMA DE AGRESIÓN VERBAL DE CARÁCTER SEXISTA EN EL INTERIOR DE LA ARQUEOLOGÍA PERUANA? RESPUESTA MASCULINA

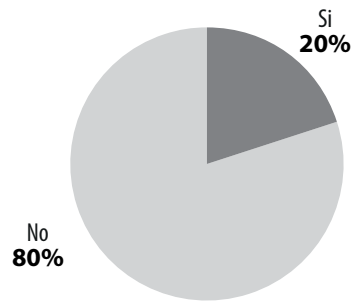

FIGURA 3. En el primer recuadro se observa la respuesta femenina, mientras que en el segundo, la respuesta masculina en relación con haber sido víctimas de agresión verbal de corte sexista.

Dentro de la encuesta se planteó un bloque de preguntas de opción múltiple, que apuntaron a abordar el tema del hostigamiento sexual como problemática al interior de la arqueología peruana. La primera preguntaba si se consideraba que el hostigamiento sexual se presenta al interior de la comunidad arqueológica. El $80 \%$ de los entrevistados respondió de forma positiva. Un $90,9 \%$ reafirmó su preocupación sobre el tema cuando se les preguntó si consideraban que el hostigamiento sexual debería ser un tema abordado al interior de nuestra comunidad.

La tercera interrogante tuvo como objetivo recuperar parte de las experiencias sociales de las mujeres ${ }^{12}$. Para ello, se pidió a las personas participantes que marcasen si habían vivido alguna de las distintas formas de violencia de género tomadas en consideración en la Ley $30220^{13}$.

12 Virginia Guzmán y Patricia Portocarrero (1992, p. 27) nos plantean que analizar la vida individual de las mujeres tiene varias ventajas: permite articular las trayectorias y las representaciones y romper con la dicotomía objetividad/subjetividad. Asimismo, hace posible descubrir la multiplicidad de la experiencia femenina y las presiones que enfrenta, así como las distintas vías que toman las mujeres para afirmarse como individuos. De esta forma, se ponen en debate la existencia de relaciones de género que se expresan en la forma como son vividas y a nivel grupal o individual.

13 La actual Ley Universitaria del Perú, que sirve de sustento legal para los actuales reglamentos de prevención y sanción del hostigamiento sexual exigidos por la Superintendencia Nacional de Educación Superior Universitaria (Sunedu), que a la vez están amparados por la Ley 29430. 


\section{ACCIONES QUE TIPIFICAN COMO VIOLENCIA DE GÉNERO. RESPUESTA FEMENINA}

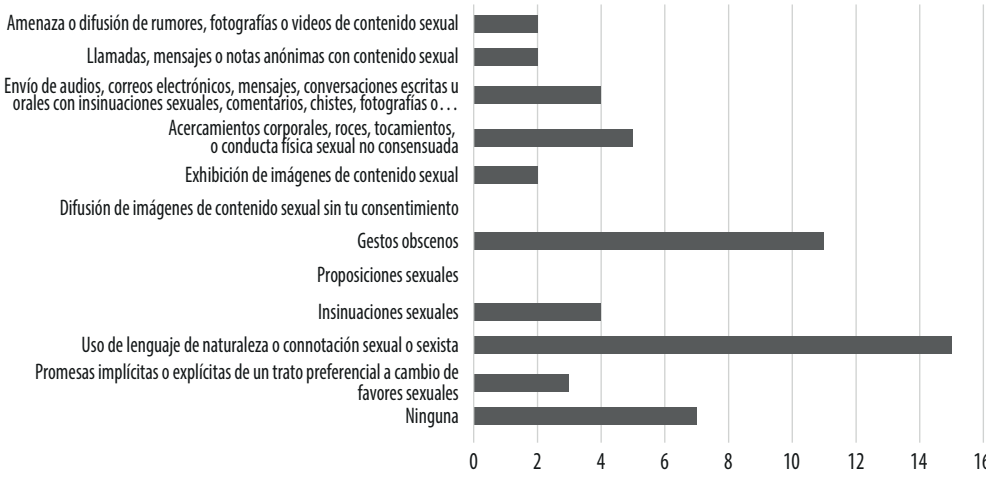

FIGURA 4. Resultados de la encuesta de las mujeres sobre acciones que tipifican como violencia de género.

En lo que respecta a la información vertida por la población femenina, un $25,9 \%$ declaró no haber vivido ninguna de las acciones que, de acuerdo con nuestras distintas leyes y normativas, son calificadas como una forma de hostigamiento sexual. En lo que se refiere a las víctimas femeninas, se puedo determinar que las dos formas más frecuentes de violencia son el uso de lenguaje de naturaleza o connotación sexual o sexista y el uso de gestos obscenos, seguidos en un segundo plano por las insinuaciones sexuales y los acercamientos corporales, roces, tocamientos, o conductas físicas sexuales no consensuadas (figura 4).

Por otra parte, este trabajo también ha considerado conveniente presentar las respuestas emitidas por la población masculina. A diferencia de las mujeres, se ha identificado que un $48,1 \%$ de los varones encuestados niegan haber vivido alguna de estas situaciones. Sin embargo, reportan haber sido víctimas del uso de lenguaje de naturaleza o connotación sexual o sexista, gestos obscenos e insinuaciones (figura 5).

\section{ACCIONES QUE TIPIFICAN COMO VIOLENCIA DE GÉNERO. RESPUESTA MASCULINA}

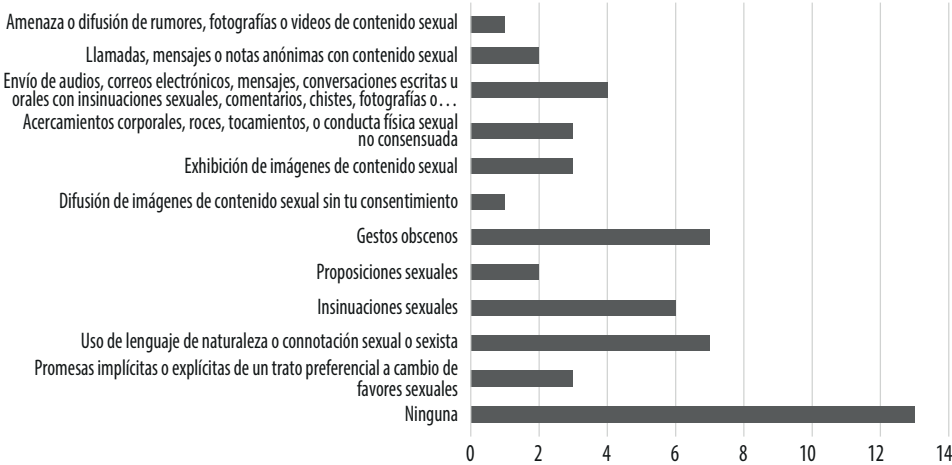

FIGURA 5. Resultados de la encuesta de las mujeres sobre acciones que tipifican como violencia de género. 
¿CONSIDERAS QUE LAS MUJERES EXPERIMENTAN LA FORMACIÓN Y PRÁCTICA ARQUEOLÓGICA DE IGUAL FORMA QUE LOS HOMBRES? RESPUESTA FEMENINA

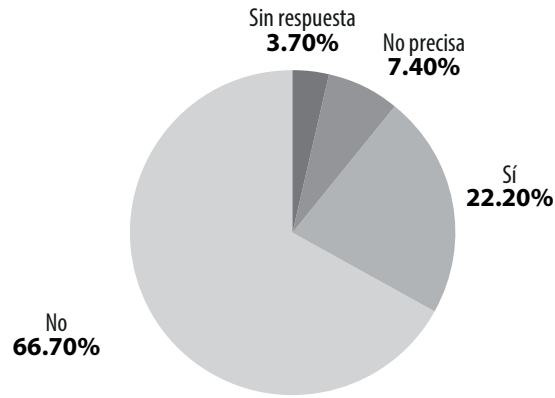

FIGURA 6. Respuesta femenina sobre la pregunta: «¿Considera que las mujeres experimentan la formación y práctica arqueológica de igual forma que los hombres?».

A manera de cierre reflexivo para la encuesta «Hablemos de arqueología» se diseñó la siguiente pregunta: «¿Consideras que las mujeres experimentan la formación y práctica arqueológica de igual forma que los hombres?». Las respuestas no fueron de opción múltiple, por lo cual nos permitió en algunos casos conocer un poco más a detalle la percepción que tienen algunas personas al interior de la comunidad arqueológica peruana; y que posteriormente se nutrirán con algunos de los testimonios que se obtuvieron de la campaña \#MeTooArqueología.

Del total de la población femenina encuestada, el 66,7\% consideró que las mujeres no experimentan formación y practica arqueológica de igual forma que sus pares masculinos. A ello se puede sumar un $11,1 \%$ que duda sobre si las experiencias al interior de la arqueología peruana se dan de forma equitativa e igualitaria (figura 6).

En lo que se refiere a la respuesta masculina, esta se encontró equitativamente polarizada entre un $50 \%$ que asegura que las experiencias de aprendizaje y practica arqueológica no son igualitarias. En contraste, un $40 \%$ considera que hombres y mujeres están en las mismas condiciones al interior de la comunidad arqueológica peruana, a lo que se adiciona un $10 \%$ que no sabe precisar su respuesta (figura 7 ). 
¿CONSIDERAS QUE LAS MUJERES EXPERIMENTAN LA FORMACIÓN Y PRÁCTICA ARQUEOLÓGICA DE IGUAL FORMA QUE LOS HOMBRES? RESPUESTA MASCULINA

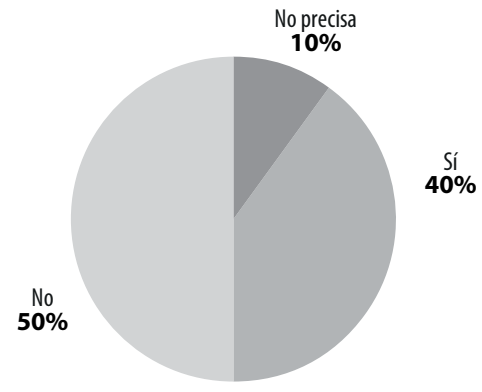

FIGURA 7. Respuestas masculinas sobre la pregunta: «¿Considera que las mujeres experimentan la formación y práctica arqueológica de igual forma que los hombres?».

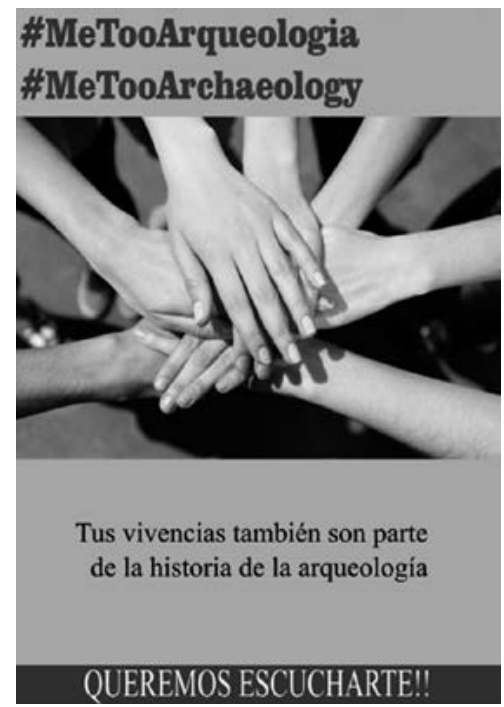

FIGURA 8. Póster de la campaña \#MeTooArqueología. Recuperado de https://www.facebook.com/Mujeresarqueologia/photos/a.350797665756219/350797685756217/?type=3\&theater

Entre algunas de las impresiones vertidas en relación con la igualdad de experiencias entre arqueólogas y arqueólogos a nivel educativo y laboral hemos rescatado las siguientes:

«No. Las mujeres tenemos la condición de ser mujeres y tener que aguantar comentarios sobre nuestra profesionalidad en excavaciones o en el campo, ya que hasta ahora la arqueología había estado 
dirigida por hombres, cosa que va a cambiar con más mujeres dedicándose a ello».

(Ind. femenino, 21-24 años, Universidad de San Marcos)

«No, debido a que mucho del trabajo de fuerza de campo se cree que solo es para hombres»

(Ind. masculino, 21-24 años, Universidad Católica)

«No [experimentamos la arqueología de igual forma], porque nos consideran "débiles" a la hora del trabajo de campo».

(Ind. femenino, 21-24 años, Universidad Nacional Pedro Ruiz Gallo)

«No del todo, muchas de las mujeres que conozco prefieren puestos o labores que no les acarreen un gran esfuerzo físico, y creo que parte de la práctica y formación del arqueólogo es ensuciarse las manos y trabajar duro. Pero en cuanto a lo demás me parece que no hay distinción, se nos dan las mismas oportunidades, incluso a veces más a ellas, pero en general la experimentamos de igual forma».

(Ind. masculino, 18-20 años, Universidad de San Marcos)

«No, están expuestas constantemente a situaciones incómodas y de agresión que comprometen su bienestar físico y emocional, así como en su desempeño profesional».

(Ind. masculino, 31-35 años, Universidad de San Marcos)

«No, debido a que mucho del trabajo de fuerza de campo se cree que solo es para hombres».

(Ind. masculino, 21-24 años, Universidad Nacional de Trujillo)

«Actualmente me parece más un tema inclinado al dinero más que al género».

(Ind. Masculino, 21-24 años, Universidad Nacional de Trujillo)

Finalmente, en lo que respecta a los testimonios recopilados por medio dela campaña \#MeTooArqueología, se hicieron pormedio delos formularios de la plataforma GoogleDrive perteneciente al «Simposio mujeres del pasado y del presente: una visión desde la arqueología peruana», que fue compartido por las redes sociales del evento (figura 8). Los testimonios fueron recabados entre febrero y mayo de 2019, con un total de 25 participantes.

Al analizar los testimonios vimos adecuado buscar recurrencias. De esa forma, se pudo identificar los espacios de agresión, el tipo de violencia 
y la posición jerárquica de los agresores al interior del campo laboral de la arqueología.

La gran mayoría de las situaciones denunciadas ocurrieron durante las temporadas de excavación en el trabajo de campo. En segundo lugar, se mencionaron las áreas de laboratorio o gabinete $y$, finalmente, las aulas universitarias.

«Me tocaba la pierna cada vez que subía a su camioneta en temporada de campo» $(1 / 2 / 2019,19: 55)$.

«En la universidad que un profesor me diga que no puedo sacar más nota que mi pareja, a pesar [de] que mi examen es mejor» (7/2/2019, 7:45).

En lo que respecta a las formas de violencia vividas por las arqueólogas, se detecta segregación de actividades laborales atribuidas a capacidades físicas o biológicas de cada sexo, desvalorización o señalamiento de limitaciones del desempeño laboral por el hecho de ser mujeres, el uso de lenguaje sexista, gestos obscenos, tocamientos indebidos y proposiciones sexuales. Y la existencia de brechas salariales por género.

«Es raro que las mujeres sepan excavar» (1/2/2019, 11:28:26).

«Tu dedícate a gabinete, en campo no vas a poder» (1/2/2019, 11:28:26).

«Preferimos que vayan hombres [al campo], porque caminaremos mucho/usaremos la motobarrena» (1/2/2019, 22:13).

«Por ser directora de un proyecto, me querían pagar un tercio menos ( $\mathrm{S} / 800$ ) de lo que finalmente le pagaron al director ( $\mathrm{S} / 1200)$ » $(2 / 2 / 2019,21: 33)$.

El campo laboral de la arqueología (al igual que otras carreras) es un ambiente altamente jerarquizado. Esta situación lo convierte en un espacio propicio para la vulneración de los derechos fundamentales de las personas que en él laboran. El escenario se puede leer entre líneas en el siguiente testimonio:

«luego [de haberme negado a mantener un encuentro sexual] en el trabajo de gabinete comenzó a hostigarme pidiéndome trabajos extras de un día para el otro (antes él estaba muy feliz de mi productividad como practicante...) y, peor aún, poniendo en duda mi capacidad intelectual y responsabilidad en el momento de trabajar. Mi jefe [llegó] a llamarme la atención por un error cometido por él [el agresor] mismo» (5/5/2019, 16:09:49). 
A partir de los datos recogidos la mayoría de las agresiones se dieron por parte de arqueólogos que desempeñaban el trabajo de jefes de unidades de excavación, de campo o gabinete. La afectada fue una subordinada de ellos. Asimismo, se hace referencia en menor medida a acciones realizadas por arqueólogos que comparten las mismas responsabilidades laborales o por parte de los obreros o técnicos de excavación.

Vimos con preocupación que muchas arqueólogas que participaron en esta campaña señalaron haber hecho los reclamos o denuncias de lo vivido con sus superiores inmediatos (directores de proyecto). Sin embargo, no consiguieron la separación o sanción del arqueólogo que cometió la falta.

«A mi antigua jefa no le gusta que las mujeres salgan embarazadas, porque cree que lo hacemos para ganar el dinero sin trabajar. Es más, me dijeron que hable acerca de planificación familiar con mis trabajadoras» $(2 / 2 / 2019,8: 08: 18)$.

\section{Comentarios finales}

Este texto muestra un marco general sobre cómo el feminismo ha abierto las puertas para que las mujeres luchen por sus derechos al interior de la sociedad. Al mismo tiempo, motiva que estos temas formen parte de las agendas estatales de cada nación, así como de instituciones internacionales como las Naciones Unidas y sus distintas agencias asociadas.

En los últimos años, la condición de las mujeres peruanas ha progresado, de acuerdo con las lecturas del IDG, cuyas muestras a nivel de macro indican que las brechas de género se acortan. A pesar de ello, aún temas como la violencia de género sigue causando estragos en nuestra sociedad.

La violencia de género se ejerce tanto en la esfera pública y privada. Se basa en las relaciones de poder establecidas en el margo estructural de nuestras sociedades, donde las mujeres viven en constante opresión y desigualdad. Este tipo de violencia afecta a la víctima en sus relaciones familiares, interpersonales y laborales (Defensoría del Pueblo, 2018), hasta causar estragos físicos o psicológicos. Una de las formas más recurrentes que se reportan en el Perú es el hostigamiento sexual en los ambientes laborales (MIMP, 2017).

Investigaciones emprendidas por el Grupo Sofia nos han permitido apreciar que las desigualdades previamente descritas también se ven reflejadas en el mundo académico de las ciencias sociales. Algunas de sus expresiones son las brechas salariales, la desvalorización del conocimiento 
producido por mujeres, el estancamiento laboral, la diferenciación en la producción académica, la maternidad, por solo mencionar algunas de ellas (Alcázar y Balarin, 2018).

En esta misma línea, en lo que respecta a la arqueología, el ingreso del feminismo en la década de 1970 generó una serie de cuestionamientos en torno a la realidad que afrontaban y afrontan las mujeres al interior de sus distintas comunidades académicas. En Europa y Estados Unidos se pasó a exponer la necesidad de visibilizar los aportes de las arqueólogas en el avance de la ciencia (Berrocal, 2009, p. 29), la necesidad de democratizar las asignaciones de fondos de investigación (Gero, 1985), el papel determinante que juega la sexualidad en el desarrollo de las carreras académicas (She, 2000; Claasen,2000) y, de forma más reciente, el hostigamiento sexual (Meyers et al., 2018).

En el Perú son escasas las investigaciones que han abordado estas problemáticas (Tavera y Santana, 2018; ver también Santana, Cabrera, Arroyo y Pacheco en este volumen), a pesar de la importancia que se le ha otorgado en distintos foros académicos. Los datos aquí recabados nos han dado unas primeras luces acerca de la violencia de género al interior de nuestra comunidad.

La población con la que se trabajo tuvo una presencia equitativa de ambos sexos, así como diversidad de poblaciones etarias provenientes de distintos espacios de formación universitaria. Consideramos ello como un punto de partida positivo en relación con la información obtenida.

Los datos y testimonios obtenidos evidencian y confirman la existencia de una dominación masculina al interior de la arqueología peruana, la cual se refleja en el señalamiento de experiencias diferenciadas de hombres, mujeres y diversidades al interior de la comunidad arqueológica peruana, y a través de los testimonios en torno a la segregación de las mujeres en la atmósfera laboral a partir de diferencias anatómicas y físicas. A ello se suma el reconocimiento de un ambiente hostil hacia la mujer y la ocurrencia de vivencias identificadas como violencia de género.

Es evidente que existen diversas formas de violencia de género; las más recurrentes son el uso de lenguaje de naturaleza o connotación sexual o sexista, la realización de gestos obscenos y las insinuaciones sexuales. En este punto es importante detenernos a analizar que en una primera instancia, cuando se preguntó a las encuestadas si habían recibido algún tipo de agresión verbal de corte sexista al interior de la arqueología peruana, solo lo reportó el $37 \%$ de ellas, porcentaje que se elevó a un 55,55\% en una segunda modalidad de pregunta. Esta situación nos hace preguntarnos cuál fue la razón del cambio de porcentaje; si está vinculado a una 
necesidad encubrimiento de la situación por parte de la víctima para no mostrar signos de debilidad o si corresponde a un proceso de normalización de la situación.

No deseamos dejar de lado el registro de violencia reportado por los varones encuestados, quienes han presentado una tasa mayor al 50\%. Es necesario señalar que en algunos países las tasas de violencia ejercida por mujeres a hombres llegan a ser casi equiparables a la registrada de forma inversa (Castro, 2016, p. 349). Sin embargo, se debe considerar que «la violencia que ejercen las mujeres no forma parte de un sistema más general de dominación y control, como sí ocurre con la violencia que ejercen los hombres sobres las mujeres» (Gondolf, 2014 en Castro, 2016). Consideramos que estas evidencias nos sugieren un alto grado de hostilidad en las dinámicas internas de la comunidad, así como una normalización de estas acciones como formas de interacción natural que no acarrean ninguna responsabilidad.

Los testimonios provenientes de la campaña \#MeTooArqueología han permitido conocer de forma más detallada las experiencias de vida de las mujeres al interior de la arqueología, e identificar los espacios y formas de hostigamiento sexual que ocurren al interior de nuestra comunidad. Resalta la redundancia de casos en los cuales los agresores tienen la posición de jefes o supervisores de las labores desempeñadas por las arqueólogas, así como la poca sensibilidad de los responsables de proyecto para afrontar este tipo de situaciones y ejercer las sanciones correspondientes.

Es claro que la violencia a la que fueron expuestas sucede en un marco de desigualdad y opresión de género, que, más allá de brindar los espacios de apoyo y acompañamiento a las víctimas, tiende a revictimizar a la persona y hacerla presa de sus miedos. Todo ello impacta en el desempeño laboral y en la vida misma de las afectadas.

Finalmente, consideramos que esta acotada recopilación nos abre una pequeña puerta sobre las experiencias de vida de las mujeres al interior de la arqueología peruana, y se convierte en un llamado a reflexionar sobre la realidad en la que estamos inmersas. Los resultados ponen a luz la necesidad de ampliar la muestra de estudio, a fin de realizar un análisis más detallado sobre las circunstancias bajo las cuales ocurren estas situaciones. Consideramos que se hace imperante que las universidades y los espacios laborales como el Ministerio de Cultura y las diversas entidades relacionadas con el ejercicio arqueológico (Coarpe) establezcan campañas de sensibilización sobre el hostigamiento sexual. Todo ello en aras de repensar las masculinidades que se ejerzan y la búsqueda de dinámicas sanas y justas al interior de la arqueología peruana. 


\section{Agradecimientos}

Este texto no hubiera sido posible sin el desprendimiento de las personas que participaron de la encuesta «Hablemos de arqueología». A las arqueólogas que decidieron desprenderse de sus historias y compartirlas en el \#MeTooArqueología, muchas gracias por su confianza. Espero que este texto les dé voz a su sentir. A Rosamar Tavera, por su comprensión. Espero poder compensarte el tiempo. A Lady Santana, por ser mi compañera en este viaje. A Henry, por estar allí para empujar el carrito juntos.

\section{Contribuciones}

La totalidad de este texto fue producido por Carito Tavera Medina.

\section{Fuentes de financiamiento}

Esta investigación fue hecha con fondos propios de la autora.

\section{Conflicto de intereses}

La autora declara que no existen conflictos de intereses.

\section{REFERENCIAS BIBLIOGRÁFICAS}

Alcázar, L. y Balarin, M. (2018). ¿Cómo abordar el estudio de las inequidades de género en el mundo académico? En L. Alcázar y M. Balarin (Eds.), Desigualdad en la academia. Mujeres en las ciencias sociales peruanas. Lima: Grupo Sofia

Arrieta de Guzmán, T. (2018). Sobre el pensamiento feminista y la ciencia. Letras, 89(130), p. 51-78.

Anderson, J. (2014) Prejuicios, paradigmas y poder: explicar (y cambiar) la posición de las mujeres en las ciencias sociales peruanas. En S. Vargas (Ed.), Bajo el radar de Sofía. Oportunidades y barreras de las profesionales en el Perú. Lima: Instituto de Estudios Peruanos:

Andreo, G. y Guardia, S. (2002). Historia de las mujeres en América Latina. Murcia: Universidad de Murcia.

Berrocal, M. (2009). Feminismo, teoría y práctica de una arqueología científica. Trabajos de Prehistoria, 66(2), pp. 25-43.

Castro, R. (2016). Violencia de género. En H. Moreno y E. Alcántara (Eds.), Conceptos clave en los estudios de género. Volumen 1. Ciudad de México: Universidad Autónoma de México.

Conkey, M. (2005). Dwelling at the margins, actions at the intersection? Feminist and Indigenous archaeologies. Archaeologies, 1(1), pp. 9-80. 
Conkey, M y Wylie A. (1998). Doing Archaeology as a feminist. Advanced seminar at the School for American Research. Santa Fe, Nuevo México.

Claassen, C. (2000). Homophobia and women archaeologists. World Arcaheology, 32(2), pp. 173-179.

Defensoría del Pueblo. (2018). Aproximación a la problemática del hostigamiento sexual laboral contra mujeres. Supervisión a ministerios, gobiernos regionales y Poder Judicial. (Informe 007-2018-DP/ADM). Recuperado de https://www.defensoria.gob.pe/wp-content/uploads/2018/07/ Informe-de-Adjuntia-007-2018-DP-ADM-II.pdf

Díaz-Andreu, M. (2013). Epílogo: últimas reflexiones y nuevas propuestas. En H. Tantaleán y C. Astuhuamán (Eds.), Historia de la arqueología en el Perú del siglo XX (pp. 603-616). Lima: IFEA-IAR.

Gero, J. (1985). Socio-politics and he woman at home ideology. American Antiquity, 50(2), pp. 342-350.

Gero, J. (1994). Excavation bias and the woman-at-home ideology. Recuperado de https://anthrosource.onlinelibrary.wiley.com/doi/abs/10.1525/ ap3a.1994.5.1.37

Gero, J. y Conkey, M. (Eds.) (1991). Engendering Archaeology. Women and Prehistory. Oxford: Blackewell.

González Moreno, J. (2012). La participación de las mujeres en la producción científica en ciencia y tecnología en el Perú. Un análisis de los trabajos publicados en ECIPERU, Revista del encuentro científico internacional (2006-2011). Recuperado en https://guzlop-editoras.com/web_des/edu01/pld0359. pdf

Guzmán, V. y Portocarero, P. (1992) Construyendo diferencias. Lima: Flora Tristán.

Hunnicutt, G. (2009). Varieties of patriarchy and violence against women. Resurrectting "patriarchy» as theorical tool. Violence Against Women, 16, pp.553-573.

Instituto Nacional de Estadística e Informática (2016). Perú: Brechas de género 2016. Avances hacia la igualdad de mujeres y hombres. Recuperado de https://www.inei.gob.pe/media/MenuRecursivo/publicaciones_digitales/Est/Lib1388/Libro.pdf

Jaiven, A. (2016). Feminismos. En H. Moreno y E. Alcántara (Eds.). Conceptos clave en los estudios de género: Volumen 1. Ciudad de México: Universidad Autónoma de México.

Mannarelli, M. E. (2018). La domesticación de las mujeres. Patriarcado y género en la historia peruana. Lima: La Siniestra Ensayos. 
Meyers, M., Horton, E., Boudreaux, E., Carmody, S., Wright, A. y Dekle, V. (2018). The context and consequences of sexual harassment in Southeastern archaeology. Advances in Archaeological Practice, 6(4), pp. 275-287.

Ministerio de la Mujer y Poblaciones Vulnerables (2017). Violencia basada en género: Marco conceptual para las políticas públicas y la acción del Estado. Recuperado de https://www.mimp.gob.pe/files/direcciones/dgcvg/ mimp-marco-conceptual-violencia-basada-en-genero.pdf

Organismo de Evaluación y Fiscalización Ambiental (2018). Alza tu voz, ante el acoso, acusa, Reporte \#02. Recuperado de http://www.oefa.gob. pe/?wpfb_dl=29990

Renfrew, C y Bahn, P. (2001) Archaeology: Theories, Methods and Practice. Londres: Thames \& Hudson.

Rosas, L. (2019). Género y mujeres en la historia del Perú. Del hogar al espacio público. Lima: Fondo Editorial de la Pontificia Universidad Católica del Perú.

She (2000). Sex and a Career. World Archaeology, 32(2), pp. 166-172

Tavera, C. y Santana, L. (11 de octubre del 2018). Desigualdades impresas: un primer paso para el estudio de la mujer en la arqueología peruana. I Jornada de Estudios de Género y Mujeres en la Historia del Perú.

Vargas, V., Orving, H. y Trapazo R. (2004) 25 Años de feminismo en el Perú. Historia, confluencias y perspectivas. Recuperado de http://www2.congreso.gob.pe/sicr/cendocbib/con4_uibd.nsf/C08CBB7DF991A3FF05257B17 00675D74/\$FILE/BVCI0003574.pdf

Vargas, S. (2014). Bajo el radar de Sofía: Oportunidades y barreras de las profesionales en el Perú. Lima: Instituto de Estudios Peruanos.

Zegarra, F. (1999). Mujeres y género en la historia del Perú. Lima: CendocMujer.

Wylie, A. (2007). Doing archaeology as a feminist: Introduction. Journal of Archaeological Method and Theory, 14(3), pp. 209-2016. 